\title{
Tumor angiogenesis, macrophages and mast cell microdensities in endometrioid endometrial carcinoma
}

\author{
CRISTIANA SIMIONESCU ${ }^{1}$, CLAUDIU MĂRGĂRITESCU ${ }^{1}$, ALEX STEPAN ${ }^{1}$, \\ DANIEL PIRICI $^{2}$, RALUCA CIUREA $^{1}$ and NICOLAE CERNEA ${ }^{3}$ \\ Departments of ${ }^{1}$ Pathology, ${ }^{2}$ Histology and ${ }^{3}$ Obstetrics and Gynecology, \\ University of Medicine and Pharmacy of Craiova, Craiova 200349, Romania
}

Received January 15, 2013; Accepted June 5, 2013

DOI: $10.3892 / \mathrm{ol} .2013 .1412$

\begin{abstract}
The present study aimed to observe and compare the values of microvessel density (MVD), mast cell microdensity (McMD) and macrophage microdensity (MphMD) in intratumoral areas compared with the advancing edges, and to assess any correlations between these values and the degree and stage of the neoplasia. The cases of 52 patients who were diagnosed with endometrial carcinoma between 2003 and 2011 were analyzed, the majority of which were in the first stage of the disease (44 cases). Double sequential immunohistochemistry and the hot-spot counting method were used to assess the MVD (CD105 ${ }^{+}$MVD), McMD [tryptase ${ }^{+}\left(\operatorname{Try}^{+}\right)$McMD] and MphMD (CD68 ${ }^{+}$MphMD) densities. The $\chi^{2}$ test, paired Student's t-test and the Pearson correlation index were used to assess the significance of the results. A weak correlation was observed at the advancing edge only, between CD105 ${ }^{+}$MVD and $\mathrm{Try}^{+} \mathrm{McMD}(\mathrm{P}=0.039)$. No significant differences were identified in the analysis of $\mathrm{CD}_{105^{+}} \mathrm{MVD}$, Try ${ }^{+} \mathrm{McMD}$ and $\mathrm{CD}^{+} 8^{+} \mathrm{MphMD}$, but wide variations in their distribution were observed. Depending on the tumor stage, CD105 ${ }^{+}$MVD exhibited an intratumoral, indirect correlation with Try ${ }^{+} \mathrm{McMD}$ for stage IA $(\mathrm{P}=0.026)$ and II $(\mathrm{P}=0.013)$ tumors. $\mathrm{CD} 105^{+} \mathrm{MVD}$ presented an indirect correlation with $\mathrm{CD} 68^{+} \mathrm{MphMD}$ in stage IB tumors $(\mathrm{P}=0.016)$ and at the advancing edge for well-differentiated tumors $(\mathrm{P}=0.027)$. An analysis of the correlation between $\mathrm{CD}^{2} 8^{+} \mathrm{MphMD}$ and $\mathrm{Try}^{+} \mathrm{McMD}$ indicated that the intratumoral levels of $\mathrm{CD}^{+} 8^{+} \mathrm{MphMD}$ were directly proportional with the Try ${ }^{+} \mathrm{McMD}$ values in well-differentiated $(\mathrm{P}=0.005)$ and stage II $(\mathrm{P}=0.012)$ tumors, while at the front of the invasion, this correlation was indirect $(\mathrm{P}=0.010)$ in stage II tumors. In endometrioid endometrial carcinoma (EEC), angio-
\end{abstract}

Correspondence to: Professor Cristiana Simionescu, Department of Pathology, University of Medicine and Pharmacy of Craiova, 2-4 Petru Rareş Street, Craiova 200349, Romania

E-mail: csimionescu2004@yahoo.com

Key words: endometrial carcinoma, tumor angiogenesis, macrophages, mast cells genesis is at its most active at the advancing edge of the tumor, where mast cells play a pro-angiogenic role.

\section{Introduction}

Almost $97 \%$ of all reported endometrial cancers are classed as carcinomas, which encompass a heterogenous group of tumors that display a range of biological, morphological and pathological characteristics (1-4).

It is well known that tumor transformation and progression do not represent singular events, but involve several steps and complex interactions, including the inflammatory process and angiogenesis, associated with tumors that are establishing a growth-favorable local microenvironment. Macrophages, as a major component of the tumor microenvironment, release growth factors that affect tumor cells or the endothelium of tumor vessels and promote the recruitment of secondary inflammatory cells, including mast cells and neutrophils (5).

In addition, tumor-associated macrophages (TAMs) appear to play a key role in tumor angiogenesis by modulating tumor growth and invasion (6-13). However, mast cells are also known to be able to synthesize and release strong angiogenic cytokines (14), such as tryptase. Tryptase has been reported to be released by mast cells in areas of angiogenesis and also to play a significant role in neovascularization (15).

The aim of the present study was to assess tumor angiogenesis quantified by microvessel density (MVD), mast cell density (McMD) and macrophage density (MphMD), and to assess any putative correlations these factors may have with the tumor stage and grade in patients with endometrioid endometrial carcinoma (EEC).

\section{Material and methods}

Patients. The cases of 52 patients who were diagnosed with endometrial carcinoma between 2003 and 2011 in the Laboratory of Pathology from the County Emergency Clinical Hospital Craiova (Craiova, Romania) were analyzed. Furthermore, five samples of normal endometrium and myometrium were used as controls. The biological material was obtained from hysterectomy fragments that had been fixed in $10 \%$ buffered neutral formalin and then classically processed for paraffin embedding and hematoxylin-eosin staining. 
Table I. Panel of antibodies used for the immunohistochemistry.

\begin{tabular}{|c|c|c|c|c|}
\hline Antibody & Clone, manufacturer & Dilution & Antigenic retrieval & External positive control \\
\hline CD105 (endoglin) & Polyclonal, Thermo Scientific & $1: 50$ & Citrate, $\mathrm{pH} 6$ & Kidney \\
\hline Mast cell tryptase & AA1, Dako & $1: 100$ & Citrate, $\mathrm{pH} 6$ & Skin \\
\hline CD68 & KP1, Dako & $1: 50$ & Citrate, $\mathrm{pH} 6$ & Skin \\
\hline
\end{tabular}

Thermo Scientific (Motortech, Timisoara, Romania); Dako (Redox Bucharest, Romania).

The grade and stage of the neoplasias were used as the morphoclinical parameters and were classified according to the guidelines by the World Health Organization (WHO) (16). In Romania at present, there is no National Register for patients who have been diagnosed with endometrial adenocarcinoma. Approval for the present study was obtained from the ethics committee of the University of Medicine and Pharmacy of Craiova, and written informed consent was obtained from all patients.

Immunohistochemistry. The panel of antibodies that were used for the immunohistochemical analysis are presented in Table I. The analysis of the morphology, topography and density of the elements of interest was based on sequential double immunohistochemical reactions using the CD105, mast cell tryptase and CD68 antibodies successively. To detect the first antibody, an LSAB2-HRP amplification and detection system (K0675; Dako, Redox Bucharest, Romania) was used with DAB chromogen development (3467; Dako). Detection of the second antibody was performed using a species-specific LSAB2-AP System (K0674; Dako) and chromogen Vulcan Fast Red (FR805S; Biocare Medical, MedicaRom, Bucharest, Romania). An avidin-biotin blocking step was included between the two procedures to mask any available biotin that remained following the first round of the reaction (X0590; Dako). Positive and negative external controls, which omitted the primary antibodies, were also included (data not shown).

The immunohistochemical analysis allowed the visualization of the blood vessels (stained with CD105), mast cells (stained with tryptase) and macrophages (stained with CD68) in all investigated cases. Thus, the McMD, MphMD and MVD in the tumor were determined. Microvascularity was defined as a single endothelial cell or group of endothelial cells that was positive for CD105 and formed a visible lumen that was clearly separated from the adjacent microvessels.

Microdensity measurement and image acquisition. The microdensity measurements were performed intratumorally and at the advancing edge. The intratumoral area was defined as stromal tissue that contained two or more neoplastic islands, and the invasion front/advancing edge was considered to be a positive structure located on the limit of the tumor-free tissue.

Image acquisition was performed using a Nikon Eclipse E600 microscope, equipped with the Lucia 5 image analysis software (Nikon, Apidrag, Bucharest, Romania).

The microdensities of the elements of interest were determined using the method described by Weidner et al (17). The slides were initially scanned at x100 magnification to identify the areas with the highest densities. The quantification was reported as the average value (area or number) for 10 hot-spot fields, with an area of $0.7386 \mathrm{~mm}^{2}$, using a $20 \mathrm{X}$ objective lens.

To assess the reproducibility of the method, the specimens were counted independently by two observers with a correction factor $\kappa$-value of 0.07 .

Statistical analysis. For the statistical analysis, a paired Student's t-test, one way ANOVA and Pearson's correlation index were performed using SPSS 10 software (SPSS, Inc., Chicago, IL, USA). The values were reported as mean \pm standard deviation. The data averages of the groups were used in order to create data groups and classes for further tests.

\section{Results}

Normal tissue immunoprofile. The $\mathrm{CD} 105^{+} \mathrm{MVD}$ values in the normal uterus specimens were higher in the endometrium (7.6 \pm 2.1 ; x200 magnification) compared with the myometrium $(4.3 \pm 1.3$; x200 magnification). The analysis of McMD in the normal uterus specimens indicated higher average values in the myometrium $(9.7 \pm 1.7 ; \times 200$ magnification) compared with the endometrium $(3.7 \pm 1.3$; x200 magnification), while MphMD had higher values in the endometrium $(93 \pm 43.7 ; \times 200$ magnification) compared with the myometrium (3.2 $\pm 1.2 ; \times 200$ magnification).

Clinical data. The average age of the patients with endometrioid carcinoma was 57.8 years (range, 43-75 years). In terms of the degree of differentiation, the well- and moderately-differentiated tumors of stages IA (17 cases) and IB (27 cases), respectively, were most prevalent, as the majority were diagnosed in the early stages.

Immunohistochemistry. The analysis of the $\mathrm{CD} 105^{+} \mathrm{MVD}$, $\mathrm{Try}^{+} \mathrm{McMD}$ and $\mathrm{CD}^{+} 8^{+} \mathrm{MphMD}$ values revealed no significant differences, but there were variations in their distribution when the data were grouped into tumor topography (intratumoral vs. advancing edge), grade and tumor stage subcategories.

The morphology and topography of the vessels that were stained using CD105 indicated the presence of numerous vascular structures, which were more concentrated at the

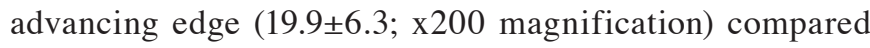

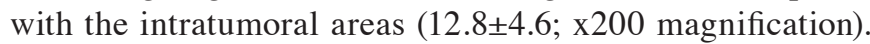
The vessels from the advancing edge had significantly larger lumens, and were more branched and dilated compared with 

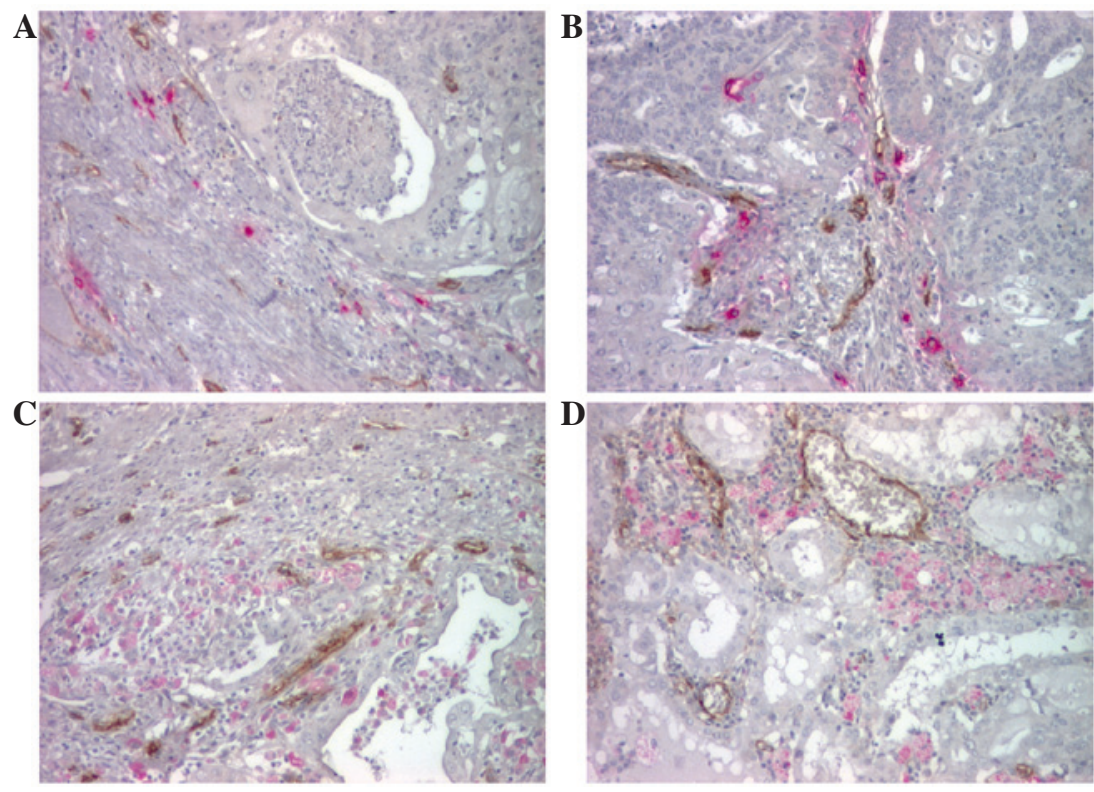

Figure 1. Endometrial carcinoma. Mast cells and microvessels from (A) the invasion front and (B) the intratumoral area; double immunohistochemistry for tryptase (AP-Fast Red; red and CD105 (HRP-DAB; brown). Macrophages and microvessels from (C) the invasion front and (D) intratumoral area; double immunohistochemistry for CD68 (AP-Fast Red; red) and CD105 (HRP-DAB; brown). Magnification, x100.

A
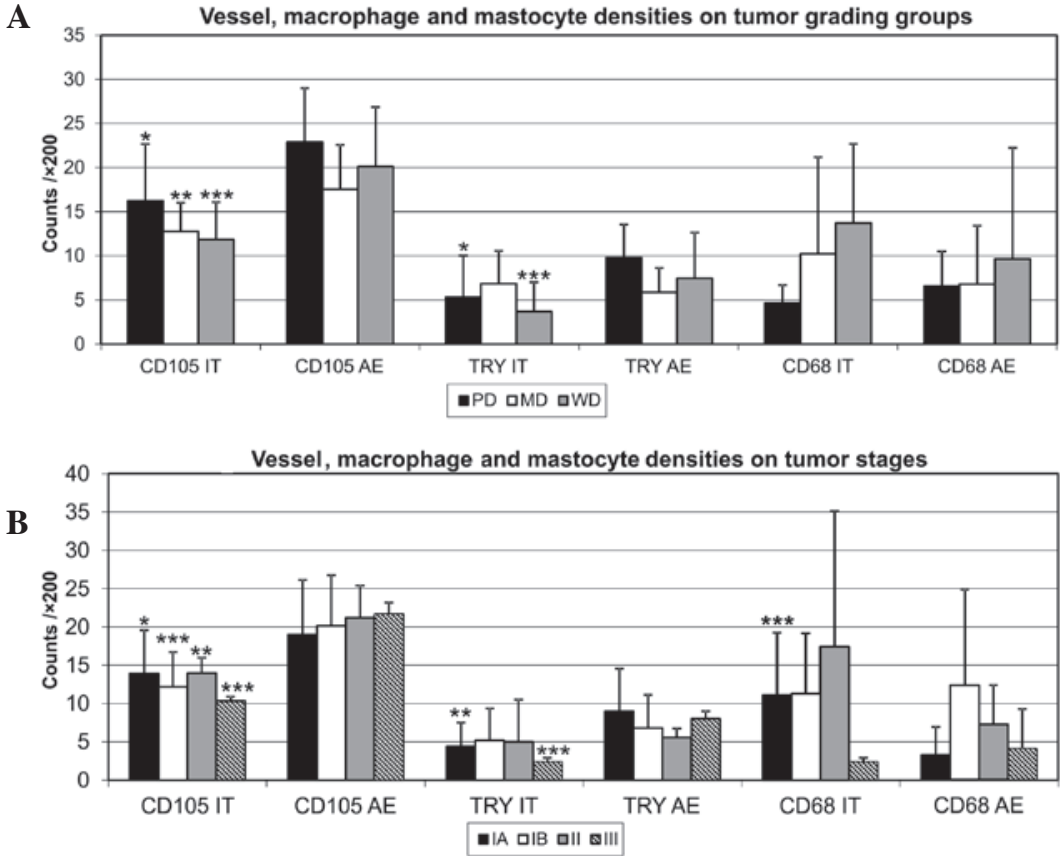

Figure 2. The distribution of vessels $\left(\mathrm{CD} 105^{+}\right)$, macrophages $\left(\mathrm{CD} 68^{+}\right)$and mast cells (TRY) depending on tumor (A) differentiation degree and (B) stage. TRY, tryptase; IT, intratumoral; AE, advancing edge; WD, well-differentiated; MD, moderately-differentiated; PD, poorly-differentiated. Error bars represent standard deviation. ${ }^{*} \mathrm{P}<0.05 ;{ }^{* *} \mathrm{P}<0.01 ;{ }^{* * *} \mathrm{P}<0.001$.

the intratumoral vessels, which were less branched with a collapsed lumen (Fig. 1).

The analysis of the $\mathrm{Try}^{+}$mast cells revealed that they were present in all the studied cases, with various shapes, sizes, degrees of degranulation and aspects of mastocytoclasia. Overall, the mast cells were located mainly around the vessels (Fig. 1A and B), isolated or associated with the inflammatory site, and were more numerous at the advancing edge (7.4 $\pm 4.5 ; \times 200$ magnification) compared with the intratumoral location (4.7 \pm 3.8 ; $\times 200$ magnification).
The $\mathrm{CD} 68^{+}$macrophages were also present in all cases studied, with a dendritic morphology, abundant cytoplasm and round-oval nuclei. Variable densities of the macrophages were observed, being more numerous in the intratumoral areas (11.2 \pm 9.2 ; x200 magnification) and predominantly perivascular (Fig. 1C and D) or adjacent to areas of necrosis compared with the advancing edge of the tumor $(8.3 \pm 10.2$; x200 magnification).

The data from the immunohistochemical analysis for the mean microdensities of the morphological elements of interest, 
Table II. Correlations of MVD, McMD and MphMD in EEC with clinico-morphological investigated parameters.

\begin{tabular}{|c|c|c|c|c|c|c|c|}
\hline \multirow[b]{2}{*}{ Parameter } & \multicolumn{3}{|c|}{ Tumoral degree } & \multicolumn{4}{|c|}{ Tumoral stage } \\
\hline & WD & MD & PD & IA & IB & II & III \\
\hline No. & 30 & 13 & 9 & 17 & 27 & 5 & 3 \\
\hline \multicolumn{8}{|l|}{ MVD } \\
\hline IT & $11.8 \pm 4.2$ & $12.8 \pm 3.2$ & $16.2 \pm 6.4$ & $13.9 \pm 5.6$ & $12.2 \pm 4.5$ & $14.0 \pm 2.0$ & $10.3 \pm 0.6$ \\
\hline $\mathrm{AE}$ & $20.1 \pm 6.7$ & $17.5 \pm 5.0$ & $22.8 \pm 6.1$ & $19.0 \pm 7.2$ & $20.1 \pm 6.6$ & $21.2 \pm 4.2$ & $21.7 \pm 1.5$ \\
\hline P-value & $1.938 \times 10^{-7}$ & 0.004 & 0.019 & 0.014 & $1.875 \times 10^{-6}$ & 0.004 & 0.000 \\
\hline \multicolumn{8}{|l|}{ McMD } \\
\hline IT & $3.7 \pm 3.3$ & $6.8 \pm 3.7$ & $5.3 \pm 4.7$ & $4.4 \pm 3.1$ & $5.2 \pm 4.2$ & $5.0 \pm 5.5$ & $2.3 \pm 0.6$ \\
\hline $\mathrm{AE}$ & $7.5 \pm 5.2$ & $5.8 \pm 2.8$ & $9.7 \pm 3.8$ & $9.0 \pm 5.6$ & $6.8 \pm 4.4$ & $5.6 \pm 1.1$ & $8.0 \pm 1.0$ \\
\hline P-value & 0.000 & 0.221 & 0.020 & 0.002 & 0.088 & 0.408 & 0.000 \\
\hline \multicolumn{8}{|l|}{ MphMD } \\
\hline IT & $13.7 \pm 8.9$ & $10.2 \pm 10.9$ & $4.6 \pm 2.0$ & $11.0 \pm 8.2$ & $11.3 \pm 7.9$ & $17.4 \pm 17.7$ & $2.3 \pm 0.6$ \\
\hline $\mathrm{AE}$ & $9.6 \pm 12.6$ & $6.8 \pm 6.6$ & $6.5 \pm 3.9$ & $3.2 \pm 3.6$ & $12.3 \pm 12.5$ & $7.2 \pm 5.2$ & $4.0 \pm 5.2$ \\
\hline P-value & 0.075 & 0.169 & 0.108 & 0.000 & 0.358 & 0.125 & 0.305 \\
\hline
\end{tabular}

EEC, endometrioid endometrial cancer; MVD, microvessel density; MphMD, macrophage microdensity; McMD, mast cell microdensity; IT, intratumoral; AE, advancing edge; WD, well-differentiated; MD, moderate-differentiated; PD, poor-differentiated. P-values were obtained by Student's t-test. All data, with the exception of No., are presented as mean \pm standard deviation.

depending on the grade and tumor stage, are presented in Table II.

Clinico-morphological correlations. According to the histological grade of the analyzed endometrioid carcinomas, the Student's t-test indicated that $\mathrm{CD} 105^{+}$MVD for all degrees of differentiation and $\mathrm{Try}^{+} \mathrm{McMD}$ for well- and poorly-differentiated tumors had significantly lower intratumoral values compared with the advancing edge (Fig. 2A).

The analysis of $\mathrm{CD}_{105^{+}} \mathrm{MVD}, \mathrm{Try}^{+} \mathrm{McMD}$ and CD68 ${ }^{+}$MphMD, depending on the tumor stage using a Student's t-test, revealed that the values of CD105+ MVD for all stages and of $\mathrm{Try}^{+} \mathrm{McMD}$ for stages IA and III, were lower intratumorally compared with the advancing edge (Fig. 2B). In contrast, the CD68 ${ }^{+} \mathrm{MphMD}$ values in stage IA tumors showed significant differences and were higher intratumorally compared with the advancing edge.

Overall, using Pearson's test, CD105+ MVD indicated a weak correlation with $\operatorname{Try}^{+} \operatorname{McMD}[\mathrm{r}(50)=0.287, \mathrm{P}=0.039]$ at the advancing edge of the tumor.

When considering the data grouped into tumor stages, Pearson's test indicated that $\mathrm{CD} 105^{+} \mathrm{MVD}$ exhibited an intratumoral, indirect correlation with $\mathrm{Try}^{+} \mathrm{McMD}$ stage IA $[\mathrm{r}(15)=-0.538, \mathrm{P}=0.026]$ and $\mathrm{II}[\mathrm{r}(3)=-0.951, \mathrm{P}=0.013]$ tumors CD105 ${ }^{+}$MVD also presented an indirect correlation with $\mathrm{CD} 68^{+} \mathrm{MphMD}$ at the advancing edge for well-differentiated forms $[\mathrm{r}(28)=-0.403, \mathrm{P}=0.027]$ as well with lesions from stage IB tumors $[\mathrm{r}(25)=-0.458, \mathrm{P}=0016]$.

Pearson's test showed that $\mathrm{CD}^{+} 8^{+} \mathrm{MphMD}$ varied in direct correlation with $\mathrm{Try}^{+} \mathrm{McMD}$ in well-differentiated $[\mathrm{r}(28)=0.502, \mathrm{P}=0.005]$ and stage II $[\mathrm{r}(3)=0.952, \mathrm{P}=0.012]$ tumors. In contrast, at the advancing edge of the stage II tumors, this correlation was indirect $[\mathrm{r}(3)=-0.959, \mathrm{P}=0.010]$.

\section{Discussion}

Angiogenesis and inflammation have numerous common pathways, as they are biological processes that are closely associated with cancer. Angiogenesis is critical for the continuous growth of tumors and the development of metastases (18). The initiation of angiogenesis is primarily regulated by the balance between pro- and anti-angiogenic factors, and represents an early and essential event in tumor development and progression $(19,20)$. A significant role in this process appears to be played by the interaction between tumor cells and the tumor microenvironment, which indirectly contributes to the induction of angiogenesis.

Macrophages and mast cells are pivotal inflammatory cells in the tumor stroma, and are present in the majority of malignant neoplasms. In the second half of the 1990s, a correlation was identified between mast cells and angiogenesis in various malignancies (21). Mast cell tryptase was shown to be a potent angiogenic factor (15). Furthermore, several studies have demonstrated the association between the intensity of tumor vascularization and macrophage infiltration in numerous cancers, including endometrial cancer (22), suggesting that TAMs increase the angiogenic potential of tumors by producing pro-angiogenic factors, including vascular-endothelial growth factor (VEGF) (23).

In the present study, certain differences were identified between the distribution of $\mathrm{CD}_{105^{+}} \mathrm{MVD}$ and Try ${ }^{+} \mathrm{McMD}$ in relation to the tumor grade and stage. CD105 ${ }^{+} \mathrm{MVD}$ for tumors of all degrees of differentiation, and $\mathrm{Try}^{+} \mathrm{McMD}$ in well- and poorly-differentiated tumors, had significantly lower intratumoral values compared with the advancing edge. Depending on the tumor stage, CD105+ MVD for all stages and $\mathrm{Try}^{+} \mathrm{McMD}$ for stages IA and II were lower intratumor- 
ally compared with the advancing edge. Overall, the Pearson correlation test indicated the existence of a weak correlation

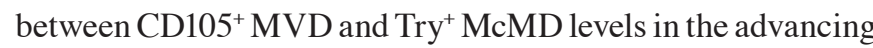
edge. In contrast, the analysis of the correlation with regard to the tumor stage between CD105+ MVD and Try ${ }^{+}$McMD indicated an intratumoral, indirect correlation for stage IA and II tumors. Although angiogenesis, quantified by $\mathrm{CD} 105^{+} \mathrm{MVD}$ and $\mathrm{Try}^{+} \mathrm{McMD}$, was more active at the advancing edge, only a weak statistical correlation was observed between the two parameters. This would argue in favor of the pro-angiogenic effect of mast cells in the advancing edge of endometrial carcinomas.

Certain studies have indicated the existence of a correlation between MVD and McMD and the degree of differentiation of endometrial carcinomas (24), while others have recorded its absence (25-27).

Ribatti et al (24) report that endometrial carcinoma angiogenesis, measured by the number of $\mathrm{CD} 31^{+}$microvessels, is strongly correlated with the number of $\mathrm{Try}^{+}$mast cells, with poorly-differentiated tumors containing a higher number of vessels compared with well-differentiated tumors. In contrast, no correlation was observed in other studies between the density of C-kit ${ }^{+}(26)$ or $\operatorname{try}^{+}(27)$ mast cells or the number of $\mathrm{CD} 1^{+}$microvessels and the tumor grade. Gosku et al (25) reported a more intense vascularity in the tumor stroma and myometrium of high-grade endometrial cancers compared with low-grade tumors, and also that try ${ }^{+}$mast cells do not increase in parallel with the histological grade of tumors. The lack of correlation between mast cell density, angiogenesis and the histological tumor grade seem to suggest that mast cells are not a significant prognostic factor in tumor progression (25).

Data obtained from the literature on the correlation between mast cells density and tumor stage are also contradictory. Ribatti et al (24) reported that angiogenesis and try ${ }^{+}$ mast cell number increases with tumor progression. Similarly, Cinel et al (26) indicated a statistically significant correlation between a high density of $\mathrm{C}-\mathrm{kit}^{+}$mast cells and the presence of myometrial invasion, with high levels in 54\% of the analyzed cases, $94 \%$ of which were also associated with myometrial invasion. In contrast, other studies have reported no correlation between Try ${ }^{+}$McMD and myometrial invasion $(25,27)$.

In the present study, similar to the $\operatorname{Try}^{+} \mathrm{McMD}$ analysis, the test for the CD68 ${ }^{+} \mathrm{MphMD}$-grouped data showed certain differences with regard to the tumor stage. The levels of $\mathrm{CD} 8^{+} \mathrm{MphMD}$ were higher intratumorally than in the advancing edge in stage IA tumors. With regard to the association between $\mathrm{CD} 105^{+} \mathrm{MVD}$ and CD68 ${ }^{+} \mathrm{MphMD}$, the existence of certain indirect correlations at the advancing edge for well-differentiated forms, as well as for stage IB tumors, were identified. Overall, the lack of correlation between $\mathrm{CD}_{105^{+}} \mathrm{MVD}$ and $\mathrm{CD} 8^{+} \mathrm{MphMD}$ values would indicate a minor role of these cells in the angiogenesis process.

In the case of macrophages, data from various studies with regard to the correlation between the tumor grade and stage are also controversial. Espinosa et al (28) reported high $\mathrm{CD} 68^{+}$macrophage infiltration in high-grade endometrioid carcinomas and the presence of a greater number of CD $31^{+}$ vessels compared with low-grade endometrioid tumors. Hashimoto et al (22) reported that TAMs from tumor nests and stroma were significantly increased in high-grade tumors. A univariate analysis by Soeda et al (29) observed that a statistically significant correlation existed between the number of intratumoral TAMs and the tumor grades defined by the International Federation of Gynecology and Obstetrics (FIGO), and also between the number of intratumoral TAMs and MVD. Another study has shown that the moderate or strong expression of VEGF is significantly associated with a high MVD and an increased number of $\mathrm{CD}^{+} 8^{+}$macrophages for aggressive tumor subgroups (30). The literature reports a correlation between macrophages and myometrial invasion, but not as a prediction factor for prognosis $(22,28,29)$.

Certain studies have provided statistically significant results for macrophage infiltration and myoinvasive vs. non-myoinvasive tumors $(28)$ and the depth of myometrial invasion $(22,29)$, suggesting that tumor angiogenesis is triggered and enhanced by stromal macrophages, which regulate the progression of endometrial carcinoma (28). Ohno et al (31) reported that only the $\mathrm{CD} 8^{+}$macrophages from within the areas of necrosis were associated with the clinical stage and level of myometrial invasion, while Soeda et al (29) identified this correlation for the $\mathrm{CD} 68^{+}$macrophages in the intratumoral and advancing edge areas.

In the present study, $\mathrm{Try}^{+} \mathrm{McMD}$ and $\mathrm{CD} 68^{+} \mathrm{MphMD}$ at the intratumoral level were correlated in well-differentiated and stage II forms. At the advancing edge, there was an indirect correlation in stage II tumors. Although there were no significant correlations between McMD and MphMD, an indirect correlation was observed between the two parameters in the stage II tumors at the advancing edge, and a direct correlation intratumorally. This would emphasize the dual agonist-antagonist roles of the two cell populations in the processes of angiogenesis and tumor progression in endometrial carcinoma.

The existence of conflicting results in the literature is most likely to be caused by the large variation in tumor types and stages, the location of the inflammatory cells and the methods used to label the inflammatory cells and blood vessels, in addition to the lack of a standardized assessment of angiogenesis or the inflammatory cells $(25,32)$. A significant limitation is the variation in the methods for reporting the topography of the mast cells and macrophages (31). In addition, labeling the vessels for CD31 $(24,25,27,28)$ or CD105 $(33,34)$, the mast cells for tryptase $(24,25,35)$, toluidine blue (27) or C-kit (26) and the macrophages for CD68 (29-31) or CD163 (28) may cause discrepancies.

In conclusion, angiogenesis was observed to be more intense in the advancing edge, where it showed a weak correlation with $\operatorname{Try}^{+} \mathrm{McMD}$. The correlation between $\mathrm{CD} 8^{+} \mathrm{MphMD}$ and angiogenesis was indirect and only identified at the intratumoral level in stage IA and II tumors. A direct correlation existed between $\operatorname{Try}^{+} \mathrm{McMD}$ and $\mathrm{CD} 68^{+} \mathrm{MphMD}$ at the intratumoral level in well-differentiated and stage II tumors, while in the advancing edge, this correlation was indirect and only identified in stage II tumors. The present data require a quantification of angiogenesis and McMD and MphMD in endometrial carcinomas, in order to determine the prognosis and future development of alternative therapies that may target these denominators, with potential benefits for patients. 


\section{References}

1. Bokhman JV: Two pathogenetic types of endometrial carcinoma Gynecol Oncol 15: 10-17, 1983.

2. Sherman ME: Theories of endometrial carcinogenesis: a multidisciplinary approach. Mod Pathol 13: 295-308, 2000.

3. Lax SF: Molecular genetic pathways in various types of endometrial carcinoma: from a phenotypical to a molecular-based classification. Virchows Arch 444: 213-223, 2004.

4. Bansal N, Yendluri V and Wenham RM: The molecular biology of endometrial cancers and the implications for pathogenesis, classification, and targeted therapies. Cancer Control 16: 8-13, 2009.

5. Theoharides TC and Conti P: Mast cells: the Jekyll and Hyde of tumor growth. Trends Immunol 25: 235-241, 2004.

6. Crowther M, Brown NJ, Bishop ET and Lewis CE: Microenvironmental influence on macrophage regulation of angiogenesis in wounds and malignant tumors. J Leukoc Biol 70 478-490, 2001.

7. Kataki A, Scheid P, Piet M, Marie B, Martinet N, Martinet Y and Vignaud JM: Tumor infiltrating lymphocytes and macrophages have a potential dual role in lung cancer by supporting both host-defense and tumor progression. J Lab Clin Med 140: 320-328, 2002.

8. Lewis CE and Pollard JW: Distinct role of macrophages in different tumor microenvironments. Cancer Res 66: 605-612, 2006.

9. Murdoch C, Giannoudis A and Lewis CE: Mechanisms regulating the recruitment of macrophages into hypoxic areas of tumors and other ischemic tissues. Blood 104: 2224-2234, 2004.

10. Murdoch C, Muthana M and Lewis CE: Hypoxia regulates macrophage functions in inflammation. J Immunol 175: 6257-6263, 2005.

11. Condeelis $\mathbf{J}$ and Pollard JW: Macrophages: obligate partners for tumor cell migration, invasion, and metastasis. Cell 124: 263-266, 2006.

12. Dirkx AE, Oude Egbrink MG, Wagstaff J and Griffioen AW: Monocyte/macrophage infiltration in tumors: modulators of angiogenesis. J Leukoc Biol 80: 1183-1196, 2006.

13. Pollard JW: Tumour-educated macrophages promote tumour progression and metastasis. Nat Rev 4: 71-78, 2004.

14. Ribatti $\mathrm{D}$ and Crivellato $\mathrm{E}$ : The controversial role of mast cells in tumor growth. Int Rev Cell Mol Biol 275: 89-131, 2009.

15. Blair RJ, Meng H, Marchese MJ, Ren S, Schwartz LB, Tonnesen MG and Gruber BL: Human mast cells stimulate vascular tube formation. Tryptase is a novel, potent angiogenic factor. J Clin Invest 99: 2691-2700, 1997.

16. Silverberg SG, Kurman RJ, Nogales F, Mutter GL, Kubik-Huch RA and Tavassoli FA: Tumors of the uterine corpus. Epithelial tumors and related lesions. In: WHO Classification of Tumors: Pathology and Genetics of Tumors of the Breast and Female Genital Organs. Tavassoli FA and Devilee P (eds). IARC Press, Lyon, France, pp221-232, 2003.

17. Weidner N, Carroll PR, Flax J, Blumenfeld W and Folkman J: Tumor angiogenesis correlates with metastasis in invasive prostate carcinoma. Am J Pathol 143: 401-409, 1993.

18. Folkman J: What is the evidence that tumors are angiogenesis dependent? J Natl Cancer Inst 82: 4-6, 1990.

19. Hanahan D and Folkman J: Patterns and emerging mechanisms of the angiogenic switch during tumorigenesis. Cell 86: 353-364, 1996.

20. Bergers $G$ and Benjamin LE: Tumorigenesis and the angiogenic switch. Nat Rev Cancer 3: 401-410, 2003.
21. Ribatti D, Vacca A, Marzullo A, Nico B, Ria R, Roncali L and Dammacco F: Angiogenesis and mast cell density with tryptase activity increase simultaneously with pathological progression in B-cell non-Hodgkin's lymphomas. Int J Cancer 85: 171-175, 2000.

22. Hashimoto I, Kodama J,Seki N,Hongo A, Miyagi Y, Yoshinouchi M and Kudo T: Macrophage infiltration and angiogenesis in endometrial cancer. Anticancer Res 20: 4853-4856, 2000.

23. Gargett CE, Lederman F, Heryanto B, Gambino LS and Rogers PA: Focal vascular endothelial growth factor correlates with angiogenesis in human endometrium. Role of intravascular neutrophils. Hum Reprod 16: 1065-1075, 2001.

24. Ribatti D, Finato N, Crivellato E, Marzullo A, Mangieri D, Nico B, Vacca A and Beltrami CA: Neovascularization and mast cells with tryptase activity increase simultaneously with pathologic progression in human endometrial cancer. Am J Obstet Gynecol 193: 1961-1965, 2005.

25. Goksu Erol AY, Tokyol C, Ozdemir O, Yilmazer M, Arioz TD and Aktepe F: The role of mast cells and angiogenesis in benign and malignant neoplasms of the uterus. Pathol Res Pract 207: 618-622, 2011.

26. Cinel L, Aban M, Basturk M, Ertunc D, Arpaci R, Dilek S and Camdeviren $\mathrm{H}$ : The association of mast cell density with myometrial invasion in endometrial carcinoma: a preliminary report. Pathol Res Pract 205: 255-258, 2009.

27. Pansrikaew P, Cheewakriangkrai C, Taweevisit $M$, Khunamornpong S and Siriaunkgul S: Correlation of mast cell density, tumor angiogenesis, and clinical outcomes in patients with endometrioid endometrial cancer. Asian Pac J Cancer Prev 11: 623-626, 2010.

28. Espinosa I, José Carnicer M, Catasus L, Canet B, D'angelo E, Zannoni GF and Prat J: Myometrial invasion and lymph node metastasis in endometrioid carcinomas: tumor-associated macrophages, microvessel density, and HIF1A have a crucial role. Am J Surg Pathol 34: 1708-1714, 2010.

29. Soeda S, Nakamura N, Ozeki T, Nishiyama H, Hojo H, Yamada H, Abe M and Sato A: Tumor-associated macrophages correlate with vascular space invasion and myometrial invasion in endometrial carcinoma. Gynecol Oncol 109: 122-128, 2008.

30. Salvesen HB and Akslen LA: Significance of tumour-associated macrophages, vascular endothelial growth factor and thrombospondin-1 expression for tumour angiogenesis and prognosis in endometrial carcinomas. Int J Cancer 84: 538-543, 1999.

31. Ohno S, Ohno Y, Suzuki N, Kamei T, Koike K, Inagawa H, Kohchi C, Soma G and Inoue M: Correlation of histological localization of tumor-associated macrophages with clinicopathological features in endometrial cancer. Anticancer Res 24: 3335-3342, 2004.

32. Erol AY and Ozdemir O: Do mast cell phenotypes play a role in concomitantly increased microvessel density and progression of non-small cell lung cancer? Hum Pathol 42: 1056-1057, 2011.

33. Saad RS, Jasnosz KM, Tung MY and Silverman JF: Endoglin (CD105) expression in endometrial carcinoma. Int J Gynecol Pathol 22: 248-253, 2003.

34. Salvesen HB, Gulluoglu MG, Stefansson I and Akslen LA: Significance of CD 105 expression for tumour angiogenesis and prognosis in endometrial carcinomas. APMIS 111: 1011-1018, 2003.

35. Ribatti D, Nico B, Finato N and Crivellato E: Tryptase-positive mast cells and CD8-positive T cells in human endometrial cancer. Pathol Int 61: 442-444, 2011. 